ERNEST BERKELEY NATIDNAL LABURATURY CONF-9803151-.

\title{
The Role of Statistics and Thermodynamics in Nuclear Multifragmentation
}

\author{
L.G. Moretto, L. Beaulieu, L. Phair, \\ and G.J. Wozniak
}

Nuclear Science Division

March 1998

Presented at the DISTRIBUTION OF THIS DOCUMENT IS URALATED International Workshop on Nuclear Matter in Different Phases and Transitions, Les Houches, France, March 31-April 10, 1998, and to be published in the Proceedings 


\section{DISCLAIMER}

This document was prepared as an account of work sponsored by the United States Government. While this document is believed to contain correct information, neither the United States Government nor any agency thereof, nor The Regents of the University of California, nor any of their employees, makes any warranty, express or implied, or assumes any legal responsibility for the accuracy, completeness, or usefulness of any information, apparatus, product, or process disclosed, or represents that its use would not infringe privately owned rights. Reference herein to any specific commercial product, process, or service by its trade name, trademark, manufacturer, or otherwise, does not necessarily constitute or imply its endorsement, recommendation, or favoring by the United States Government or any agency thereof, or The Regents of the University of California. The views and opinions of authors expressed herein do not necessarily state or reflect those of the United States Government or any agency thereof, or The Regents of the University of California.

Ernest Orlando Lawrence Berkeley National Laboratory is an equal opportunity employer. 


\section{DISCLAIMER}

Portions of this document may be illegible in electronic image products. Images are produced from the best available original document. 
LBNL-41936

\title{
The Role of Statistics and Thermodynamics in Nuclear Multifragmentation
}

\author{
L.G. Moretto, L. Beaulieu, L. Phair, and G.J. Wozniak \\ Nuclear Science Division \\ Ernest Orlando Lawrence Berkeley National Laboratory \\ University of California \\ Berkeley, California 94720
}

March 1998

This work was supported by the Director, Office of Energy Research, Office of High Energy and Nuclear Physics, Nuclear Physics Division, of the U.S. Department of Energy under Contract No. DE-AC03$76 \mathrm{SF} 00098$. 


\title{
THE ROLE OF STATISTICS AND THERMODYNAMICS IN NUCLEAR MULTIFRAGMENTATION
}

\author{
L.G MORETTO, L. BEAULIEU, L. PHAIR AND G.J. WOZNIAK \\ Nuclear Science Division, Lawrence Berkeley National \\ Laboratory, Berkeley, California 94720
}

\begin{abstract}
The natural limit of binomial reducibility in nuclear multifragmentation is observed in excitation functions for intermediate mass fragments (IMF) of a given element $Z$. The multiplicity distributions for each window of transverse energy are Poissonian. Thermal scaling is observed in the linear Arrhenius plots constructed from the average multiplicity of each element. "Emission barriers" are extracted from the slopes of the Arrhenius plots and their possible origin is discussed.
\end{abstract}

\section{Introduction}

Multiple intermediate mass fragments emission (IMF), $3 \leq Z \leq 20$, is a common decay mode in heavy-ion collisions between $20 \mathrm{~A}$ and $100 \mathrm{~A} \mathrm{MeV}[1$, 2]. It was found $[3,4,5]$ that: 1) the experimental $Z$ integrated fragment multiplicity distributions $P_{n}^{m}$ are binomially distributed in each transverse energy $\left(E_{t}\right)$ window, where $n$ is the number of emitted fragments and $m$ is the number of throws. The transverse energy $E_{t}$ is calculated from the kinetic energies $E_{i}$ of all the charged particles in an event and their polar angles $\theta_{i}$, as $E_{t}=\sum_{i} E_{i} \sin ^{2} \theta_{i} ; 2$ ) the extracted one-fragment emission probabilities $p$ give linear Arrhenius plots (i.e. excitation functions) when $\log 1 / p$ is plotted vs $1 / \sqrt{E_{t}}$. If the excitation energy $E^{*}$ is proportional to $E_{t}$ and consequently, the temperature $T$ to $\sqrt{E_{t}}$, these linear Arrhenius plots suggest that $p$ has the Boltzmann form $p \propto \exp (-B / T)[3,4,5]$.

The charge distributions for each fragment multiplicity $n$ are also observed to be reducible to a single charge distribution and to be thermally scalable $[6,7]$.

Furthermore, the experimental particle-particle angular correlations are reducible to the individual fragment statistical angular distributions and thermally scalable [8]. 
The binomial decomposition of the IMF multiplicities reported above has been performed on the $Z$-integrated fragment multiplicities, typically associated with $3 \leq Z \leq 20$. Thus, the Arrhenius plot generated with the resulting one fragment probability $p$ is an average over a range of $Z$ values. Fortunately, it has been shown that the Arrhenius plots should survive such a $Z$ averaging, and yield an effective "barrier" (slope) dominated by the lowest $Z$ value $[3,4,5]$. However, this procedure clearly implies a substantial loss of information, and renders the binomial parameters $p$ and $m$ difficult to interpret.

As it will be shown, an analysis of the multiplicities for each fragment $Z$ value should be a considerable improvement and may solve many of these difficulties. Furthermore, it has been pointed out that a binomial distribution could be distorted by the averaging associated with the transformation $E^{*} \rightarrow E_{t}$ leading to possibly incorrect values of $m$ and $p$ [9]. However, it has been shown that, while $p$ and $m$ separately can conceivably be distorted by the transformation, the average multiplicity $\langle n\rangle=m p$ is far more resistant to the averaging process $[9,10]$. It would be useful if a way could be found of avoiding the individual extraction of $p$ and $m$ while retaining the possibility of constructing an Arrhenius plot.

In this presentation, we analyze the experimental fragment multiplicity distributions for each individual fragment $Z$ value. We show that they are Poissonian. The associated mean multiplicities for each $Z$ give linear Arrhenius plots from which the corresponding $Z$ dependent barriers can be extracted. The physical dependence of these barriers on $Z$ may shed light on the fundamental physics associated with multifragmentation, as fission barriers have done for the fission process.

\section{From binomial to Poisson distributions}

The restriction the fragment definition to a single $Z$ value has a rather dramatic effect. In Fig. 1, ratios of the variance to the mean as a function of $E_{t}$ are given for a number of $Z$ values, and for the case $Z \geq 3$ [5]. For individual $\mathrm{Z}$ values the ratios are very close to one, while for the $\mathrm{Z}$ integrated case there is a sagging at large $E_{t}$. The explanation for these features can be found by recalling that for a binomial distribution

$$
\langle n\rangle=m p ; \quad \sigma^{2}=m p(1-p) ; \quad \frac{\sigma^{2}}{\langle n\rangle}=1-p .
$$

For $p \rightarrow 0$, the ratio $\sigma^{2} /\langle n\rangle \rightarrow 1$. This is the Poisson limit. When extensive summation over $Z$ is carried out, the elementary probability $p$ increases sufficiently at the highest values of $E_{t}$ so that the Poisson distribution is replaced by the more general binomial distribution. On the other hand, the 


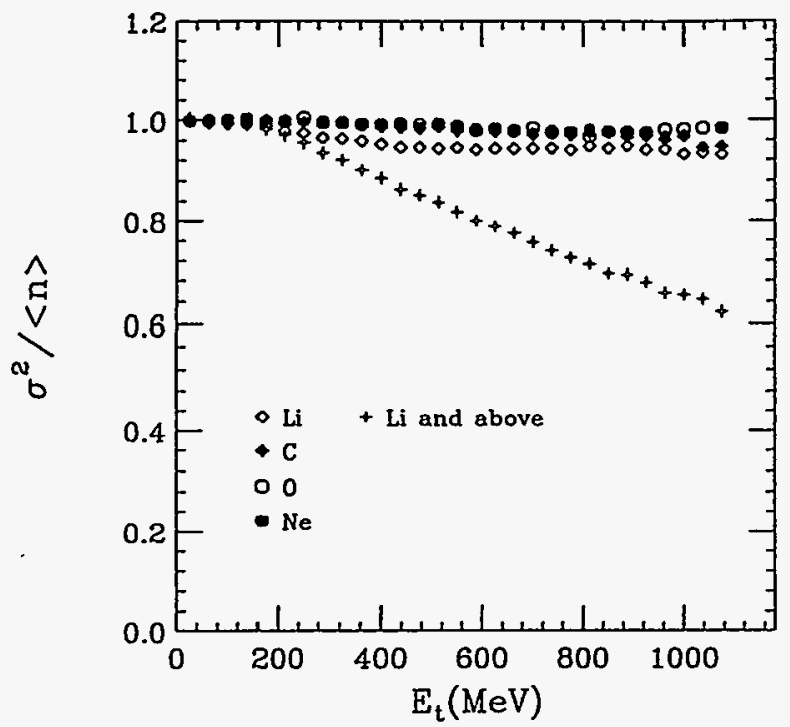

Figure 1. The ratio of the variance to the mean number of $\mathrm{Li}, \mathrm{C}, \mathrm{O}$ and $\mathrm{Ne}$ fragments (solid and open symbols) emitted from the reaction ${ }^{36} \mathrm{Ar}+{ }^{197} \mathrm{Au}$ at $E / A=110 \mathrm{MeV}$. The star symbols show the same ratio for all IMFs $(3 \leq Z \leq 20)$.

restriction to any given $Z$ value decreases the elementary probability $p$ so dramatically that the above ratio effectively remains unity at all values of $E_{t}$ and the distributions become Poissonian:

$$
P_{n}(Z)=\frac{\langle n\rangle^{n} e^{-\langle n\rangle}}{n !}
$$

where $\langle n\rangle$ is $\langle n\rangle\left(E_{t}\right)$. We show the quality of the Poisson fits to the multiplicity distribution in Fig. 2. These Poisson fits are excellent for all $Z$ values starting from $Z=3$ up to $Z=14$ over the entire range of $E_{t}$ and for all the reactions which we have studied. Thus we conclude that reducibility (we should call it now Poissonian reducibility) is verified at the level of individual $Z$ values for many different systems. Incidentally, for the $\mathrm{Xe}$ induced reactions, there is an excellent overlap of the data sets for different targets as a function of $E_{t}$. They all follow the Poisson fit to the Au target data. The probabilities $P_{n}$ and the range of $E_{t}$ increase with the increasing target mass from $\mathrm{V}$ to $\mathrm{Au}$, as they must if $E_{t}$ is a reasonable measure of the dissipated energy.

The experimental observation of Poissonian reducibility directly implies that IMF production is dominated by a stochastic process. Of course stochasticity falls directly in the realm of statistical decay, either sequential or simultaneous (see section 5.5 of ref. [5] or ref. [11]). It is less clear how it would fare within the framework of a dynamical model. 


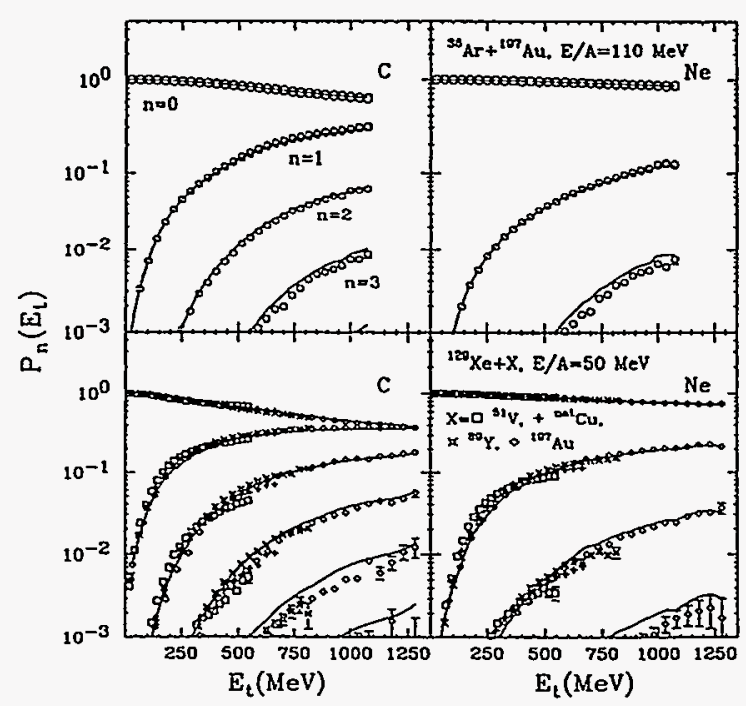

Figure 2. The excitation functions $P_{n}$ for carbon (left column) and neon emission (right column) from the reactions ${ }^{36} \mathrm{Ar}+{ }^{197} \mathrm{Au}$ at $E / A=110 \mathrm{MeV}$ (top panels) and ${ }^{129} \mathrm{Xe}+{ }^{51} \mathrm{~V},{ }^{\text {nat }} \mathrm{Cu},{ }^{80} \mathrm{Y},{ }^{197} \mathrm{Au}$ (bottom panels). The lines are Poisson fits to the gold target data.

\section{Thermal Scaling}

We now generate Arrhenius plots by plotting $\log \langle n\rangle$ vs $1 / \sqrt{E_{t}}$. Here, as in previous works, we assume that $E_{t} \propto E^{*}$ and that $E^{*} \propto T^{2}$, according to the simplest strongly degenerate Fermi gas dependence at constant volume. Of course, high excitation energies and/or lower densities can lead to deviations, which may well be looked for in the future. We expect $\langle n\rangle$; like $p$, to be of the form $\langle n\rangle=F(T, \ldots) e^{-B / T}$, where the specific form of the preexponential factor depends ultimately on whether a reaction theory or a chemical equilibrium description will prevail. We use the Arrhenius plot in the traditional spirit of evidentiating the leading $T$ dependence contained in the exponential. The top four panels of Fig. 3 give a family of these plots for four different reactions. Each family contains $Z$ values extending from $Z=3$ to $Z=14$. The observed Arrhenius plots are strikingly linear, and their slopes increase smoothly with increasing $Z$ value. One slight exception is the large $Z(\geq 10)$ data for $\mathrm{Xe}+\mathrm{Cu}$. At high $E_{t}$, the data deviate somewhat from the linear dependence observed elsewhere. For this smaller system, it is conceivable that charge conservation constraints lead to this behaviour.

The overall linear trend demonstrates that thermal scaling is also present when individual fragments of a specific $Z$ are considered. Even apart from the linearity of the Arrhenius plots, important information is already contained in the range covered by the yield of individual fragments over the 
range of $E_{t}$ shown in Fig. 3. For processes not dominated by phase space (e.g. low energy direct reactions), one expects the excitation function to depend weakly upon excitation energy. Typically the cross sections vary by factors of a few. In the present data, the mean multiplicity $\langle n\rangle$ varies with $E_{t}$ by one to two orders of magnitudes. This is a strong evidence for the involvement of the internal degrees of freedom typical of high barrier statistical decays.

The selection of fragments of individual $\mathrm{Z}$ selected leads to obvious advantages. For any given reaction, both Poissonian reducibility and thermal scaling are verifiable not just once, as in the binomial analysis, but for as many atomic numbers as are experimentally accessible. For example the $\mathrm{Ar}+\mathrm{Au}$ reaction $(E / A=110 \mathrm{MeV})$ shown in the top right panel of Fig. 3 . For this specific reaction, we can verify both reducibility and thermal scaling for 12 individual atomic numbers. Since there are $29 E_{t}$ bins, Poissonian reducibility is tested 29 times for each $Z$ value, i.e., $12 \times 29=348$ times for this reaction alone. Including all the cases shown in Fig. 3 , we have tested Poissonian reducibility 936 times. This is an extraordinary level of verification of the empirical reducibility and thermal scaling with the variable $E_{t}$.

Two added bonuses arise from this procedure.

1) The criticism has been raised that the linearity of the Arrhenius plots arises from an autocorrelation, since the complex fragments also contribute to $E_{t}$ [12]. In the present analysis this criticism can be dismissed, since each individual $Z$ contributes a vanishingly small amount to $E_{t}(\leq 5 \%)$, even in the region of maximum yields. Still, to be sure that there is no autocorrelation in Fig. 3, we have repeated the analysis, for $\mathrm{Xe}+\mathrm{Au}$ at $50 \mathrm{~A}$ $\mathrm{MeV}$, by: i) removing from $E_{t}$ the contribution of the individual $Z\left(E_{t}^{Z}\right)$ that we have selected (Fig. 3 , bottom left panel); ii) by using only the $E_{t}$ of the light charge particles, $E_{t}^{L C P}$ (Fig. 3 , bottom right panel). In both cases, the Arrhenius plots remain linear over almost the entire range of $E_{t}$ and cover 1 to 2 orders of magnitude. Quantitatively, the rate of change of the slopes with $Z$ remains the same regardless of the definition of $E_{t}$, as shown in the top panel of Fig. 4. This behaviour is expected if the slopes are related to some physical barriers.

In our attempt to avoid autocorrelation by excluding from $E_{t}$ all IMFs $\left(E_{t}^{L C P}\right)$ or the $Z$ value under investigation $\left(E_{t}^{Z}\right)$, we have introduced another kind of distortion. Excluding from $E_{t}$ all fragments of charge $Z$ to produce $E_{t}^{Z}$ necessarily requires that for those events where $E_{t}^{Z} \approx E_{t}$, the yield $n_{Z} \rightarrow 0$. This produces the visible turnover of the Arrhenius plots in the bottom panels of Fig. 3 (the same argument also applies to $E_{t}^{L C P}$ ). It has been verified experimentally that the maximum values of the new $E_{t}$ scale do indeed correspond to events in which the contribution from a given 


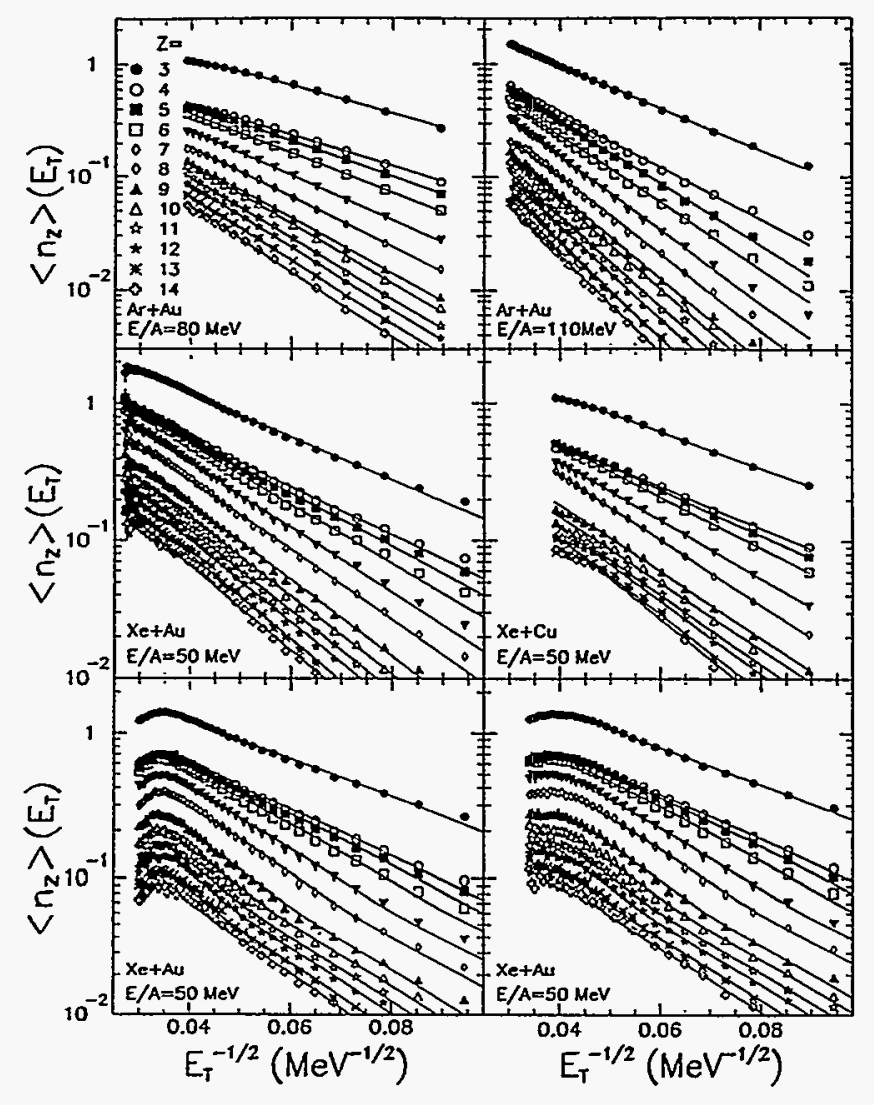

Figure 3. Middle and upper panels: The average yield per event of different elements (symbols) as a function of $1 / \sqrt{E_{t}}$. Bottom panels: The Xe+Au data at $50 \mathrm{~A} \mathrm{MeV}$ are replotted using the transverse energy of all charged particles excluding the $Z$ that we have selected, $E_{t}^{Z}$ (left), and (right) that only of the light charged particles, $E_{t}^{L C P}$. The lines are fits to the data using a Boltzmann form for $\left\langle n_{Z}\right\rangle$.

$Z$ (or all IMFs) is absent.

2) The extracted elementary probability is now $\langle n\rangle=\langle m p\rangle$ which, contrary to $p$ and $m$, is very resilient to any averaging associated with the transformation from $E^{*}$ to $E_{t}[9,10]$.

This procedure leading to Poisson distributions does not contradict binomial reducibility. To the contrary, it represents its natural limit for small values of $p$, and it expands its applicability by considering each $Z$ value individually. In going from binomial to Poissonian distributions, the price one pays is the loss of the parameter $m$. While in many ways this is a convenient result, it actually implies a loss of scale. In the time sequential interpretation of multifragmentation [5] this implies a loss of information about the time window during which multifragmentation occurs in units 


\section{THE ROLE OF STATISTICS AND THERMODYNAMICS IN NUCLEAR MULTIFRAGMENTATIOY}

of the natural channel period, or the unit time to which the elementary probability is referred. In the spatial interpretation, one loses information about the total mass of the source [5].

Poissonian reducibility and thermal scaling do not contradict recent observations regarding the role of reaction dynamics in the formation of the hot primary sources $[13,14,15,16,17,18]$. In particular, the experimental scaling is not affected by the presence of multiple sources [5] and the analysis presented here is a powerful test to establish the degree of thermalization in the late stage of the reaction. Kinematic variables seem to retain spatialtemporal information about the reaction dynamics $[14,15,16,17,18,19]$ while the associated emission probabilities seem to demonstrate, as verified nearly a 1000 times in the present work, the role of phase space in describing the decay of the sources.

\section{Z-dependent Barriers}

From the Arrhenius plots for individual atomic numbers, we can obtain the values of the slopes from Fig. 3 as a function of $Z$. The interpretation of these slopes as "emission barriers" is very tempting. If we had the correct excitation energy, rather than $E_{t}$, we could obtain the actual barriers as a function of $Z$. Unfortunately we are limited to our running variable $\dot{E}_{t}$, and to the assumption of its proportionality to $E^{*}$. However, the many linear Arrhenius plots shown here cannot be easily explained without invoking this proportionality. Therefore, with the necessary caution, we explore the possible meaning of these "barriers". A plot of these barriers as a function of $Z$ is potentially rich in information. The extracted barriers are shown in Fig. 4 (bottom panel). The barriers appear to increase linearly with $Z$ at low $Z$ values and tend to sag below linearity at higher ones.

One could wonder about the role of surface energy on the origin of these barriers. Fragments might be thought as forming by coalescence into a relatively cold and dense nuclear drop out of a hot diluted source. The appearance of a substantial surface energy for the fragment would suggest barriers proportional to $Z^{2 / 3}\left(A^{2 / 3}\right)$. If this were true, then one would expect the barrier for each $Z$ to be nearly independent of the system studied. Unfortunately, since the relation between $E_{t}$ and excitation energy is unknown, the absolute values of our barriers are also unknown. By normalizing all systems at $Z=6$ and using the $\mathrm{Xe}+\mathrm{Au}$ at $\mathrm{E} / \mathrm{A}=50 \mathrm{MeV}$ as the reference, one observes barriers that are indeed fairly independent of the system (Fig. 4, bottom panel). Another possibility is to compare the dependence of the barriers on $Z$ to that of the conditional barriers measured at low energy [20] (black dots). Their similarity with the multifragmentation barriers is dramatically illustrated. While the Coulomb-like $Z$ dependence of these 


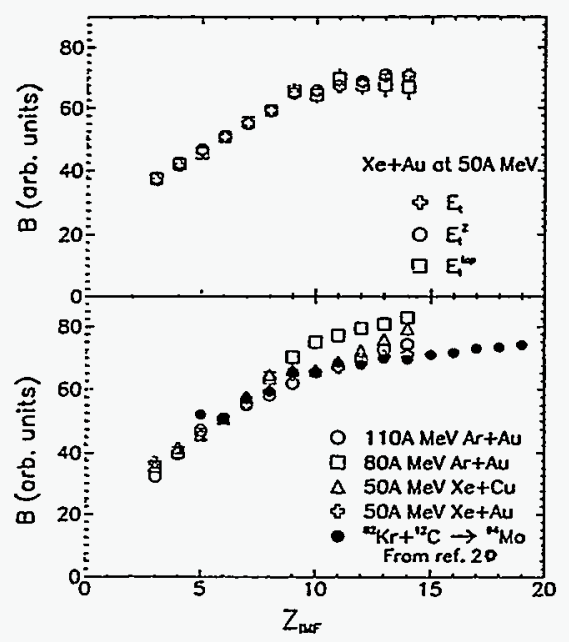

Figure 4. Top panel: Slopes of the Arrhenius plots, normalized to $Z=6$, for $\mathrm{Xe}+\mathrm{Au}$ at $50 \mathrm{~A} \mathrm{MeV}$ as a function of $Z$ using the indicated definitions of $E_{t}$. Bottom panel: The $Z$ dependent "barriers" (the slopes of the Arrhenius plots in Fig. 3). The "barriers" have been scaled relative to $Z=6$ of the $\mathrm{Xe}+\mathrm{Au}$ data. Black dots are low energy conditional barriers from ref. [20] normalized to $Z=6$ of Xe+Au.

barriers is suggestive, we should remark that these are emission barriers rather than Coulomb barriers. Thus the dominance of the Coulomb term is by no means obvious.

In conclusion, Poissonian reducibility and thermal scaling of individual fragments of a given $Z$ have been observed experimentally for several different systems at bombarding energies ranging from 50 to $110 \mathrm{MeV} /$ nucleon. The high level of verification strongly supports the stochastic/statistical nature of fragment production and provides a clear signal for source(s) thermalization in the late stage of the reaction. Slope parameters were extracted from the Arrhenius plots. The interpretation of these slopes as emission barriers, originating either from Coulomb or surface terms, or both, still needs to be explored. If the physical significance of these $Z$ dependent "barriers" must remain lamentably open, there is at least the distinct possibility that important physical information is contained therein. Data with isotopically resolved light charged particles and IMFs are needed to further investigate these phenomena.

\section{Acknowledgements}

This work was supported by the Director, Office of Energy Research, Office of High Energy and Nuclear Physics, Nuclear Physics Division of the US Department of Energy, under contract DE-AC03-76SF00098. One of us 
(L.B) acknowledge a fellowship from the National Sciences and Engineering Research Council (NSERC), Canada.

\section{References}

1. B. Borderie, Ann, de Phys. 17, 349 (1992).

2. L.G. Moretto and G.J. Wozniak, Ann. Rev. Nucl. Part. Sci. 43, 379 (1993).

3. L.G. Moretto et al., Phys. Rev. Lett. 74, 1530 (1995).

4. K. Tso et al., Phys. Lett. B 361, 25 (1995).

5. L.G. Moretto, et al., Phys. Rep. 287, 249 (1997).

6. L. Phair et al., Phys. Rev. Lett. 75, 213 (1995).

7. L.G. Moretto et al., Phys. Rev. Lett. 76, 372 (1996).

8. L. Phair et al., Phys. Rev. Lett 77, 822 (1996).

9. J. Toke et al., Phys. Rev. C 56, R1683 (1997).

10. L. Phair et al., to be published.

11. A.S. Botvina and D.H.E. Gross, Phys. Lett. B344, 6 (1995)

12. M.B. Tsang et al., Phys. Rev. Lett. 80, 1178 (1998)

13. C.P. Montoya et al., Phys. Rev. Lett 73, 3070 (1994).

14. J. Lukasik et al., Phys. Rev. C 55, 1906 (1997).

15. Y. Larochelle et al., Phys. Rev. C 55, 1869 (1997).

16. J. Toke et al., Phys. Rev. Lett. 75, 2920 (1995).

17. J.F. Lecolley et al., Phys. Lett. B 354, 202 (1995).

18. J.F. Dempsey et al., Phys. Rev. C 54, 1710 (1996).

19. D.R. Bowman et al., Phys. Rev. Lett 70, 3534 (1993).

20. K.X. Jing et al., LBNL-40779, submitted to Phys. Rev. C. 